Full length article

\title{
PI3K signaling pathways modulated white spot syndrome virus (WSSV) replication in Procambarus clarkii
}

\author{
Huijing Zhang ${ }^{\mathrm{a}, \mathrm{b}, 1}$, Xuemei Yao ${ }^{\mathrm{b}, 1}$, Yunfei Ding ${ }^{\mathrm{a}}$, Zheng Xu ${ }^{\mathrm{a}}$, Rongning Liang ${ }^{\mathrm{c}}$, Ying Zhang ${ }^{\mathrm{a}, *}$, \\ Yulong $\mathrm{Wu}^{\mathrm{a}}$, Boqing $\mathrm{Li}^{\mathrm{a}}$, Bo Guan ${ }^{\mathrm{c}, * *}$ \\ ${ }^{a}$ School of Basic Medical Sciences, Binzhou Medical University, Yantai, 264003, Shandong, China \\ ${ }^{\mathrm{b}}$ State Key Laboratory of Marine Resource Utilization in South China Sea, Hainan University, Haikou, 570228, Hainan, China \\ ${ }^{\mathrm{c}}$ Key Laboratory of Coastal Zone Environmental Processes and Ecological Remediation, Yantai Institute of Coastal Zone Research (YIC), Chinese Academy of Sciences \\ (CAS), Yantai, 264003, Shandong, China
}

\section{A R T I C L E I N F O}

\section{Keywords:}

PI3K

WSSV replication

Apoptosis

Bax

Bax inhibitor-1

Lectin

Toll

Procambarus clarkii

\begin{abstract}
A B S T R A C T
The PI3K/AKT signaling pathway is commonly exploited to regulate viral replication and affect the fate of infected cells. In the present study, a PI3K-specific inhibitor (LY294002) was employed to pretreat crayfish to evaluate the effects of PI3K/AKT signaling pathway in WSSV replication. The results showed that the WSSV copy numbers in crayfish pretreated with LY294002 were significantly lower than those in Tris- $\mathrm{HCl}$ pretreatment crayfish on the sixth and tenth day after WSSV infection. In semigranular cells, the apoptosis rates were upregulated on the third day post-WSSV infection, and a significantly lower proportion of apoptosis cells were observed in LY294002-pretreatment group. The expression level of Bax, Bax inhibitor-1 and lectin mRNA in haemocytes of crayfish were increased after WSSV infection. After the secondary stimulation with Tris- $\mathrm{HCl}$, the Bax expression level in LY294002-pretreatment crayfish was significantly higher than that of crayfish pretreated with Tris- $\mathrm{HCl}$ on the third or sixth day, but the Toll and lectin mRNA expression decreased significantly on the third, sixth and tenth day. The Bax mRNA expression levels in LY294002-WSSV group were significantly higher than those in Tris-HCl-WSSV group on the third and tenth day. The Bax inhibitor-1 mRNA expression levels in LY294002-WSSV group were significantly lower than those in Tris-HCl-WSSV crayfish on the third day. These results together indicated that the hosts PI3K/AKT signaling pathway play positive roles in WSSV replication through the balance between host cell apoptois and innate immune responses. This information is helpful to further understand the role of PI3K/AKT signaling pathway on WSSV replication in Decapoda crustaceans.
\end{abstract}

\section{Introduction}

The phosphatidylinositol-kinase (PI3K)-AKT signaling pathway regulates several key cellular functions, such as protein synthesis, glucose metabolism, cell growth and survival [1]. It is established that viral infections exploit PI3K/AKT signaling pathway to regulate viral cellular entry, ensure successful virus replication, drive viral protein expression, as well as the suppression of the host's antiviral activities [2]. Virus modulate PI3K/AKT signaling pathway to enter host cells, such as Zaire Ebola virus (ZEV), human immunodeficiency virus type 1 (HIV-1), Avian leukosis virus (ALV), human herpes viruses (HHV), cytomegalovirus (CMV) and herpes simplex virus (HSV) [3-7]. The PI3K/ AKT signaling pathway has been demonstrated to be required not only for viral cell entry but also for subsequent viral replication [6]. For example, inhibition of PI3K activation by a PI3K-specific inhibitor (LY294002) during porcine circovirus type 2 (PVC2) and respiratory syndrome virus (PRRSV) infection both led to lower virus yield, which is associated with decreased viral DNA replication and lower virus protein expression [8,9]. Influenza A virus also activated the PI3K/AKT signaling pathway to augment its efficient replication [10]. Therefore, to complete the viral life cycle, PI3K/AKT signaling pathway is commonly exploited to regulate viral replication and affect the fate of infected cells.

Apoptosis is defined as an active physiological process of cellular self-destruction, which is also utilized for viruses to evade the host immune system to ensure viral replication, viral spread, persistent infection and inflammatory responses [11]. The process of apoptosis is controlled by different ranges of cellular signaling pathways. Inhibition

\footnotetext{
* Corresponding author.

*** Corresponding author.

E-mail addresses: zhangying99g99@163.com (Y. Zhang), bguan@yic.ac.cn (B. Guan).

${ }^{1}$ These authors contributed equally to the paper.
} 
of PI3K activation with LY294002 could reduce HSV protein expression and increase the cleavage and release of caspase-3 [6]. Likewise, during largemouth Bass virus (LMBV), dengue virus (DEN) or Japanese encephalitis virus (JEV) infection, blockage of PI3K activation by LY294002 greatly enhanced virus-induced apoptosis at an early stage of infection [11-13]. Leu et al. proposed that invasion of white spot syndrome virus (WSSV) could lead to increase expression of proapoptosis proteins, membrane permeabilization of mitochondria, and cellular apoptosis [14]. Although the apoptosis has been documented to occur in several shrimps infected with WSSV, such as Litopenaeus vannamei and Penaeus monodon $[15,16]$, the PI3K/AKT signaling pathway involved in these processes still remained largely unknown.

WSSV has a broad host range of Decapoda crustaceans, such as penaeid shrimp, crab and crayfish. It is difficult to prevent and inhibit the spread of the virus due to the wide host range and easy replication, which caused huge economic losses during the last three decades [17]. However, Invertebrates have developed various defense mechanisms to recognize and eliminate the potential pathogens during the process of evolution. Recognition of non-self is the first line in mounting immune responses, and it is mediated by a group of proteins named pattern recognition receptors (PRRs). There are growing evidence that PRRs (i.e., Toll-like receptor and lectin) are closely related to virus replications in hosts $[18,19]$. Toll receptors are evolutionary ancient families of PRRs with crucial roles in invertebrate innate immune response against WSSV [20]. And lectins are one type of important PRRs that widely distribute in almost all organisms and involve in many biological processes, including cell-cell interaction, protein synthesis, and signal transduction [21]. Although PRRs involved in host immune defense responses against WSSV have been characterized, the potential biological mechanism is currently unclear. The studies of WSSV replication and host-interactions would contribute to the development management strategies for WSSV prevention and disease control.

The freshwater crayfish, Procambarus clarkii, is one of the most important commercially cultivated crustaceans in China. Based on the previous study of enrichment of the PI3K/AKT signal pathway in the regulation of viral replication, we investigated the role and mechanisms of PI3K/AKT regulation during WSSV infection in the crayfish P. clarkii. In the present study, a PI3K-specific inhibitor (LY294002) was employed to pretreat crayfish comparatively to evaluate the effects of PI3K/AKT signaling pathway in WSSV replication. Alteration of WSSV copy numbers, cell apoptosis, the apoptotic associated factors (Bax and Bax inhibitor-1) and PRRs (Toll-like receptor and lectin) were measured to elucidate the potential mechanism of PI3K/AKT signaling pathway in the crayfish $P$. clarkii against WSSV. Taken together, these results of the interaction between WSSV replication and the PI3K/AKT signaling pathway would provide new insight into the mechanism of WSSV pathogenesis in crayfish or other commercial Decapoda crustaceans.

\section{Materials and methods}

\subsection{Crayfish and virus preparation}

Crayfish $P$. clarkii (approximately $21.3 \pm 1.8 \mathrm{~g}$ each) were separately collected from aquatic markets in Yantai, Shandong Province, Weishan Lake, Shandong Province and Lianyungang, Jiangsu Province, China, and cultured in aerated water tanks with fresh water at $25 \pm 2{ }^{\circ} \mathrm{C}$ for two weeks before processing.

The shrimps with WSSV were provided from Yellow Sea Fisheries Research, Institute Chinese Academy of Fishery Sciences. WSSV was purified by differential centrifugation in shrimps. Briefly, $1 \mathrm{~g}$ of the gill tissue from the infected shrimps was homogenized in $45 \mathrm{~mL}$ of TNE buffer ( $50 \mathrm{mM}$ Tris-HCl, pH7.5, $400 \mathrm{mM} \mathrm{NaCl}, 5 \mathrm{mM}$ EDTA, pH 8.5) with $1 \mathrm{mM}$ PMSF. Thereafter, it was centrifuged at $3500 \times g$ for 5 min at $4{ }^{\circ} \mathrm{C}$, and the supernatant was then centrifuged at $30000 \times g$ for $30 \mathrm{~min}$ at $4^{\circ} \mathrm{C}$. The precipitate was resuspended in $10 \mathrm{~mL}$ TM buffer (50 mM Tris- $\mathrm{HCl}, 10 \mathrm{mM} \mathrm{MgCl}_{2}, \mathrm{pH} 7.5$ ) and then centrifuged at
Table 1

Names and sequences of primers used in this study.

\begin{tabular}{ll}
\hline Primer name & Sequence \\
\hline WSSV-F154 & CCAGTTCAGAATCGGACGTT \\
WSSV-R154 & AAAGACGCCTACCCTGTTGA \\
TaqMan probe & TCCATAGTTCCTGGTTTGTAATGTGCCG \\
Oligo (dT)-adaptor & GGCCACGCGTCGACTAGTAC(T)17 \\
Bax & \\
Pc-Bax-RT-F & TATAGTTGGCTCATTAGCAG \\
Pc-Bax-RT-R & ATACTAAGTGAAGATGACTG \\
Bax inhibitor-1 & \\
PcBI-1-RT-F & TGCCATTACATCTTGGGTTCT \\
PcBI-1-RT-R & CGACCTAATCCCATCTCAAGC \\
lectin & \\
RT-PcL-lecF & \\
RT-PcL-lecR & CTTGGTGGCTGCCTTAGAG \\
Toll & TCCTTCTCATTGTTTGTCATTC \\
Toll-F & \\
Toll-R & TCATTTGGCATCTGGCTCAC \\
18sRNA & GCAGGTGGTGGCGTTGA \\
18s RNA-RT-F & \\
$18 s$ RNA-RT-R & TCTTCTTAGAGGGATTAGCGG \\
\hline
\end{tabular}

$30000 \times g$ for $20 \mathrm{~min}$ at $4{ }^{\circ} \mathrm{C}$. Finally, the precipitate was resuspended in $1 \mathrm{~mL} \mathrm{TM}$ buffer with $0.3 \% \mathrm{NaN}_{3}$ as WSSV stock solution and stored at $-80^{\circ} \mathrm{C}$ until use.

\subsection{WSSV quantification}

The standard curve for WSSV quantification was performed according to a TaqMan real-time assay [17]. A WSSV DNA fragment of 154 bp amplified using primers WSSV-F154 and WSSV-R154 (Table 1) for real-time PCR was ligated into pMD18T-Easy vector and cloned into DH5 $\alpha$. The WSSV copy numbers of the target amplicon in the plasmid were estimated, and target amplicon in the plasmid was used to make 10-fold serial dilutions. The TaqMan real-time PCR was assayed in a PCR mixture containing $0.25 \mu \mathrm{M}$ WSSV-F154, $0.25 \mu \mathrm{M}$ WSSV-R154, $0.125 \mu \mathrm{M}$ TaqMan probe (Table 1 ) and the serial dilutions of genomic DNA in a final reaction volume of $20 \mu \mathrm{L}$. Regression of the log of WSSV copy number and the corresponding CT value was used as a standard curve for determining viral load. The viral copy number was normalized on a ng genomic DNA basis.

\subsection{Drug treatment}

A PI3K-specific inhibitors LY294002 was purchased from Cell Signaling Technology, which was dissolved in 10\% DMSO and diluted with Tris- $\mathrm{HCl}$ ( $50 \mathrm{mM}, \mathrm{pH7.5})$. Thirty crayfish were used to survey the phosphorylated AKT expression level after LY294002 treatment. Ten crayfish were injected with LY294002 $\left(0.625 \mu \mathrm{g} \cdot \mathrm{g}^{-1}, 100 \mu \mathrm{L}\right)$ or Tris$\mathrm{HCl}(100 \mu \mathrm{L})$ respectively, and the untreated crayfish were employed as the blank group. Five crayfish were randomly sampled for western blot analysis at $2 \mathrm{~h}$ post challenge.

\subsection{Western blot analysis}

The haemocytes in crayfish were lysed with $1 \mathrm{~mL}$ cell lysis buffer (50 mM Tris- $\mathrm{HCl}, 150 \mathrm{mM} \mathrm{NaCl}, 1.0 \%$ Triton X-100, $1.0 \%$ sodium deoxycholate, and $0.1 \%$ sodium dodecyl sulfate [SDS]). The protein content was measured using the BCA Protein Assay Kit (Beyotime, Shanghai, China) and $150 \mathrm{mg}$ total protein was subjected to $12 \%$ sodium dodecyl sulfate polyacrylamide gel electrophoresis and transferred to polyvinylidene difluoride membranes. The membranes were incubated with an anti-phospho-AKT (pAKT) rabbit hAb or an anti-AKT rabbit hAb (Cell Signaling Technology, Inc, USA) overnight at $4.0^{\circ} \mathrm{C}$, 
followed by incubation with anti-rabbit IgG HRP-linked antibody (Cell Signaling Technology, Inc, USA) for $1.0 \mathrm{~h}$ at room temperature. The blots were detected with an enhanced chemiluminescence detection kit according to the manufacturer's instruction (Beyotime, Shanghai, China).

\subsection{WSSV infection}

Two hundred and eighty crayfish were used in the experiment, and they were divided into seven groups. In the first group, forty crayfish were untreated and employed as blank group. In group II or III, Forty crayfish were treated with Tris-HCl $(\mathrm{pH} 7.5,100 \mu \mathrm{L})$ or WSSV stock $\left(10^{7}\right.$ copies $\mu \mathrm{L}^{-1}, 100 \mu \mathrm{L}$ ), respectively. In group IV, the forty crayfish were injected with Tris- $\mathrm{HCl}(100 \mu \mathrm{L})$ twice with $2 \mathrm{~h}$ interval. In group $\mathrm{V}$, the forty crayfish were pretreated with LY294002 $\left(0.625 \mu \mathrm{g} \cdot \mathrm{g}^{-1}, 100 \mu \mathrm{L}\right)$ firstly and then injected with Tris- $\mathrm{HCl}(100 \mu \mathrm{L})$ at $2 \mathrm{~h}$ after the first stimulation. In group VI, the crayfish were injected with Tris- $\mathrm{HCl}$ $(100 \mu \mathrm{L})$, and then received an injection of WSSV stock $\left(10^{7}\right.$ copies $\left.\mu \mathrm{L}^{-1}, 100 \mu \mathrm{L}\right)$ at $2 \mathrm{~h}$ after the first stimulation. In group VII, the forty crayfish were pretreated with LY294002 $\left(0.625 \mu \mathrm{g} \cdot \mathrm{g}^{-1}, 100 \mu \mathrm{L}\right)$, and then they were received a secondary injection of WSSV stock $\left(10^{7}\right.$ copies $\mu \mathrm{L}^{-1}, 100 \mu \mathrm{L}$ ) at $2 \mathrm{~h}$ after the fist stimulation. Afterwards, the crayfish in every group were divided into two shares, and the crayfish of one share were used to monitor the numbers of dead crayfish and the biological issue when they got moribund. In the other share, five crayfish of each group were randomly sampled on the third, sixth and tenth day after treatment to detect alteration of WSSV copy numbers, cell apoptosis rates, and the associated factors expression (Bax, Bax inhibitor-1, Toll and lectin).

\subsection{DNA extraction and real-time PCR amplification}

Total genomic DNA was extracted from crayfish treated with WSSV by using a tissue DNA Kit (OMEGA, USA) for detecting the WSSV copy numbers. The DNA concentrations were determined by optical density at OD $260 \mathrm{~nm}$ using a Genesys 5 spectrophotometer (Spectronic instrument, Inc., New York, USA). Real-time PCR was performed by using a SYBR Premix Ex TaqTM (RR820A, Takara) containing a high-performance Taq antibody and Takara Ex Taq HS. A sample of total DNA was added to a PCR mixture containing $0.25 \mu \mathrm{M}$ WSSV-F154, $0.25 \mu \mathrm{M}$ WSSV-R154 and $0.125 \mu \mathrm{M}$ TaqMan probe in a final reaction volume of $20 \mu \mathrm{L}$. The amplification program consisted of $30 \mathrm{~s}$ at $95^{\circ} \mathrm{C}$ for initial denaturation followed by 45 cycles of $5 \mathrm{~s}$ at $95^{\circ} \mathrm{C}$ for denaturation and annealing at $35 \mathrm{~s}$ at $60^{\circ} \mathrm{C}$. The WSSV copy numbers were calculated according to the method mentioned above.

\subsection{Haemocytes collection}

From each crayfish, about $0.5 \mathrm{~mL}$ hemolymph was collected into the tube containing equal volume of anticoagulant solution $(100 \mathrm{mM}$ glucose, $140 \mathrm{mM} \mathrm{NaCl}, 30 \mathrm{mM}$ trisodium citrate, $26 \mathrm{mM}$ citric acid, $10 \mathrm{mM}$ EDTA $\mathrm{Na}_{2} \cdot 2 \mathrm{H}_{2} \mathrm{O}, \mathrm{pH}$ 4.6). The hemolymph was collected and centrifuged at $800 \times g, 4^{\circ} \mathrm{C}$ for $10 \mathrm{~min}$ to collect the haemocytes. The haemocytes were divided into two shares, one of which was subjected to the measurement of cell apoptosis, and the other one was immediately used for total RNA extraction using Trizol reagent according to the manufacture's protocol (Invitrogen, USA).

\subsection{Cell apoptosis assays}

Cell apoptosis was assessed using an Annexin V-FITC Apoptosis Detection Kit (Becton Dickinson, Franklin Lakes, NJ, USA) according to the manufacturer's instructions [22]. Briefly, $1.0 \times 10^{5}$ haemocytes were mixed with $500 \mu \mathrm{L}$ Annexin Binding Buffer, $5.0 \mu \mathrm{L}$ FITC-conjugated Annexin V antibody, and $5.0 \mu \mathrm{L}$ propidium iodide. The mixture was incubated in the dark for $15 \mathrm{~min}$ at room temperature. The relative number of apoptotic cells was determined using a Beckman Coulter EPICX-XL Flow Cytometer (Beckman Coulter, Inc., Brea, CA, USA). The normal cells without induction of apoptosis were used for fluorescence compensation regulation to remove the spectral overlap.

Crayfish haemocytes were divided into three sub-populations, hyaline cells (HC), semigranular cells (SGC) and granular cells (GC), according to their discrepant values in forward scatter (FSC) and side scatters (SSC) [23]. Flow cytometry analysis of total haemocytes, HC, SGC, and GC in crayfish at different time points was performed to study apoptosis.

\subsection{Mitochondrial membrane potential $(\Delta \psi)$}

Mitochondrial membrane potential $(\Delta \psi)$ was detected using the $\mathrm{BD}^{\mathrm{TM}}$ MitoScreen Kit (BD Biosciences, USA) according to the manufacturer's instructions. Briefly, $1.0 \times 10^{6}$ haemocytes were mixed with $12.375 \mathrm{~mL} 1 \times$ Assay Buffer, $0.125 \mathrm{~mL}$ JC-1 Stock Solution, and $0.5 \mathrm{~mL}$ JC-1 Working Solution. The mixture was incubated in the dark for $15 \mathrm{~min}$ at $37^{\circ} \mathrm{C}$ in $\mathrm{CO}_{2}$ incubator. The $\Delta \psi$ evaluated by the JC- 1 polymer/monomer fluorescence ratio was determined using a $\mathrm{BD}$ FACSAria $^{\mathrm{TM}}$ III sorter (BD Biosciences, USA).

\subsection{Quantification of Bax, Bax inhibitor-1, Toll and lectin expression by quantitative real-time $R T-P C R$}

Quantitative real-time polymerase chain reaction (RT-PCR) was performed to investigate Bax, Bax inhibitor-1, Toll and lectin mRNA expression of crayfish. Total RNA was extracted from haemocytes as described above. The SYBR Green RT-PCR assay was performed in an ABI PRISM 7500 Sequence Detection System (ThermoFisher Scientific, USA). The amplifications were conducted in triplicate in $20 \mu \mathrm{L}$ using the following thermal profile: $95^{\circ} \mathrm{C}$ for $3 \mathrm{~min}$, followed by 40 cycles at $95^{\circ} \mathrm{C}$ for $15 \mathrm{~s}$ and $58{ }^{\circ} \mathrm{C}$ for $33 \mathrm{~s}$. The Bax-specific primers Pc-Bax-RT-F and Pc-Bax-RT-R, Bax inhibitor-1-specific primers PcBI-1-RT-F and PcBI-1-RT-R, Toll-specific primers Toll3-F and Toll3-R, lectin-specific primers RT-PcL-lecF and RT-PcL-lecR, and 18s-specific primers 18s RNA-RT-F and 18s RNA-RT-R (Table 1), were used to amplify the corresponding products. The 18s RNA was chosen as reference gene for internal standardization. The comparative average-cycle threshold method was used to analyze the Bax, Bax inhibitor-1, Toll and lectin mRNA expression level. The expression level of the Bax, Bax inhibitor1 , Toll and lectin gene could be calculated, and the value of which stood for an $\mathrm{n}$-fold difference relative to the calibrator [24].

\subsection{Statistical analysis}

Data are presented as mean \pm standard deviations (SD) from at least five independent experiments. All data were subjected to one-way analysis of variance. $P \leq 0.05$ was considered statistically significant.

\section{Results}

\subsection{The expression of phosphorylated AKT}

To examine the effect of a PI3K inhibitor (LY294002) on inhibiting the activity of PI3K, the expression level of phosphorylated AKT was tested through western blot analysis. The PI3K inhibitor (LY294002) was able to reduce basal AKT phosphorylation (Fig. 1). At $2 \mathrm{~h}$ after LY294002 injection, phosphorylated/total AKT was remarkably decreased and 4/5-fold $(P<0.05)$ lower than that of Tris-HCl-treatment crayfish.

\subsection{Crayfish survival rate, standard curve and WSSV quantification}

The survival rates of crayfish were equal or greater than $80 \%$ on the tenth day and no significant difference among the seven groups was 

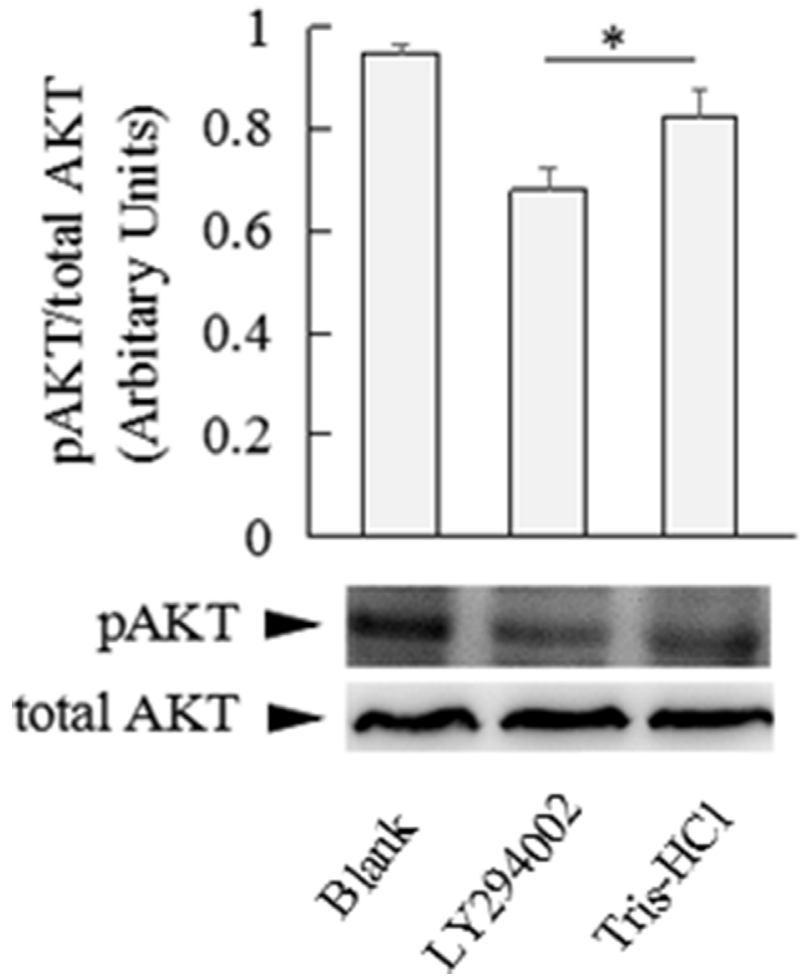

Fig. 1. Effects of a PI3K inhibitor (LY294002) on phosphorylation of AKT in crayfish. The crayfish were injected with LY294002 or Tris- $\mathrm{HCl}$ respectively, and the untreated crayfish were employed as blank group. Phosphorylated/total AKT were detected using western blot analysis. Each symbol and vertical bars represented the mean $\pm \operatorname{SD}(N=5)$. Significant differences between LY294002-treatment group and Tris-HCl-treatment group were indicated with one asterisk at $P<0.05$.

observed (data not shown). The crayfish in Tris-HCl-WSSV group got moribund on the fifth day after WSSV infection, and the moribund crayfish were observed in the LY294002-pretreatment group on the ninth day after WSSV infection.

Strong linear correlation $\left(\mathrm{R}^{2}=0.988\right)$ was obtained between CT and the WSSV copy numbers ranging from $1 \times 10^{12}$ to $1 \times 10^{1}$ in realtime PCR assay. With an optimal PCR mixture, the genomic DNA of the specimen was detected in one real-time PCR reaction, and the results showed the large dynamic range and high sensitivity of the assay (data not shown).

The WSSV copy numbers in crayfish pretreated with LY294002 were significantly lower than those in Tris- $\mathrm{HCl}$ pretreated crayfish on the sixth and tenth day post WSSV infection (Fig. 2). On the third day, the mean WSSV copy numbers in Tris-HCl-WSSV and LY294002-WSSV

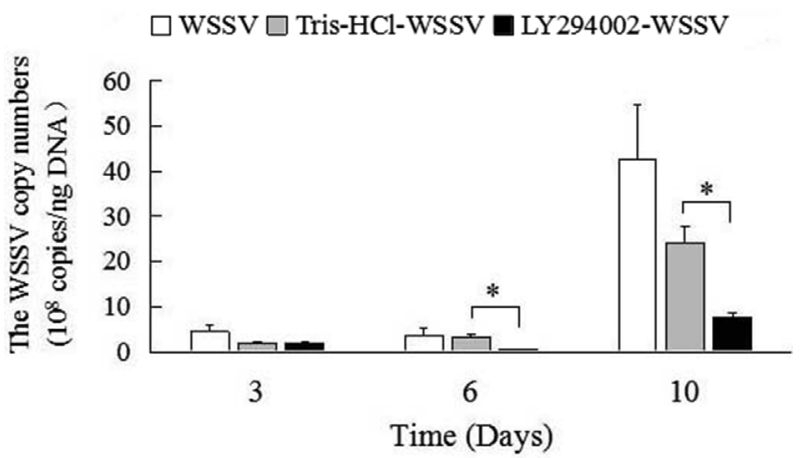

Fig. 2. The WSSV copy numbers of the crayfish in the three groups (WSSV, Tris-HClWSSV and LY294002-WSSV) on the third, sixth and tenth day. Each symbol and vertical bars represented the mean $\pm \mathrm{SD}(N=5)$. Significant differences between Tris-HCl-WSSV and LY294002-WSSV were indicated with one asterisk at $P<0.05$. group were $1.78 \times 10^{8}$ and $1.83 \times 10^{8}$ copies $\mathrm{ng}^{-1}$ DNA, respectively, and no significant difference was observed between Tris-HCl-WSSV and LY294002-WSSV group. On the sixth day, the crayfish in LY294002WSSV group represented lower WSSV loads with mean values of $0.59 \times 10^{8}$ copies $\mathrm{ng}^{-1}$ DNA, whereas $3.30 \times 10^{8}$ copies $\mathrm{ng}^{-1} \mathrm{DNA}$ WSSV copy number was detected in Tris-HCl-WSSV group. The mean WSSV copy number in Tris-HCl-WSSV group was 5.60-fold higher $(P<0.05)$ than that in LY294002-WSSV group. On the tenth day, the mean WSSV copy number of Tris-HCl-WSSV group was increased and reached $2.41 \times 10^{9}$ copies $\mathrm{ng}^{-1}$ DNA, which was 3.15 -fold higher $(P<0.05)$ than that in LY294002-WSSV group $\left(7.65 \times 10^{8}\right.$ copies $\mathrm{ng}^{-1}$ DNA).

\subsection{Apoptosis in WSSV-infected haemocytes of crayfish}

No substantial changes in apoptosis rates of total haemocytes, HC and GC were observed after infection with WSSV during the whole experiments (data not shown).

According to Annexin V-FITC apoptosis detection method in SGC, there were apoptosis rates of $12.80 \pm 3.10 \%$ and $9.87 \pm 0.68 \%$ on the third and sixth day post WSSV infection, which were 1.94-fold $(P<0.05)$ and 2.69 -fold $(P<0.01)$ higher than those post Tris-HCl stimulation, respectively (Fig. 3). When the crayfish were pretreated with LY294002, a significantly lower proportion of apoptosis cells were observed in Tris-HCl pretreatment group on the sixth and tenth day regardless whether the crayfish were infected with WSSV (Fig. 3). On the sixth day, the apoptosis rate of SGC in LY294002-Tris-HCl group was $6.80 \pm 1.00 \%$, which was remarkably lower than that in Tris-HClTris-HCl group $(P<0.05)$. Meanwhile, $5.40 \pm 0.47 \%$ of apoptotic rate was obtained in LY294002-WSSV group, and it was also lower significantly than that in Tris-HCl-WSSV group $(P<0.05)$. On the tenth day, $7.75 \pm 0.15 \%$ of apoptotic rate was obtained in LY294002Tris-HCl group. Conversely, apoptotic rate of $13.47 \pm 1.64 \%$ was found in Tris-HCl-Tris- $\mathrm{HCl}$ group. That meant a 1.74-fold $(P<0.05)$ decrease in LY294002-Tris-HCl group compared to Tris-HCl-Tris- $\mathrm{HCl}$ group. In LY294002-WSSV group, $6.17 \pm 1.23 \%$ of apoptotic rate was detected, and this rate was remarkably lower than that in Tris-HClWSSV group $(14.77 \pm 2.60 \%)(P<0.05)$.

\subsection{Mitochondrial membrane potential ( $\Delta \psi)$ in WSSV-infected haemocytes} of crayfish

No substantial changes in $\Delta \psi$ of total haemocytes, HC and GC were observed after infection with WSSV during the whole experiments (data not shown). In SGC stained with JC-1, there were lower $\Delta \psi(P<0.01)$ on the third day post WSSV infection compared to that in crayfish after Tris-HCl injection (Fig. 4). The $\Delta \psi$ in crayfish pretreated with LY294002 was higher than that in Tris-HCl-pretreatment crayfish on the third day post WSSV infection $(P<0.05)$ (Fig. 4). On the tenth day, the $\Delta \psi$ of SGC in LY294002-Tris-HCl group was remarkably higher than that in Tris-HCl-Tris- $\mathrm{HCl}$ group $(P<0.05)$ (Fig. 4).

\subsection{The Bax mRNA expression in haemocytes of crayfish}

The expression level of Bax mRNA in haemocytes of crayfish was increased on the third day after WSSV infection. The relative expression level of Bax transcript in WSSV group on the third day was up-regulated and 5.03-fold higher $(P<0.05)$ than that in Tris- $\mathrm{HCl}$ group (Fig. 5).

There was a significant increase in Bax expression level in LY294002-Tris-HCl group compared to that in Tris-HCl-Tris-HCl group on the third or sixth day (Fig. 5), and the expression levels in LY294002-Tris- $\mathrm{HCl}$ group were 11.34 -fold $(P<0.05)$ and 7.77 -fold $(P<0.01)$ higher than that in Tris-HCl-Tris- $\mathrm{HCl}$ group, respectively. The expression levels of Bax mRNA in LY294002-WSSV group were significantly higher than those in Tris-HCl-WSSV group on the third and tenth day $(P<0.05)$ (Fig. 5). 


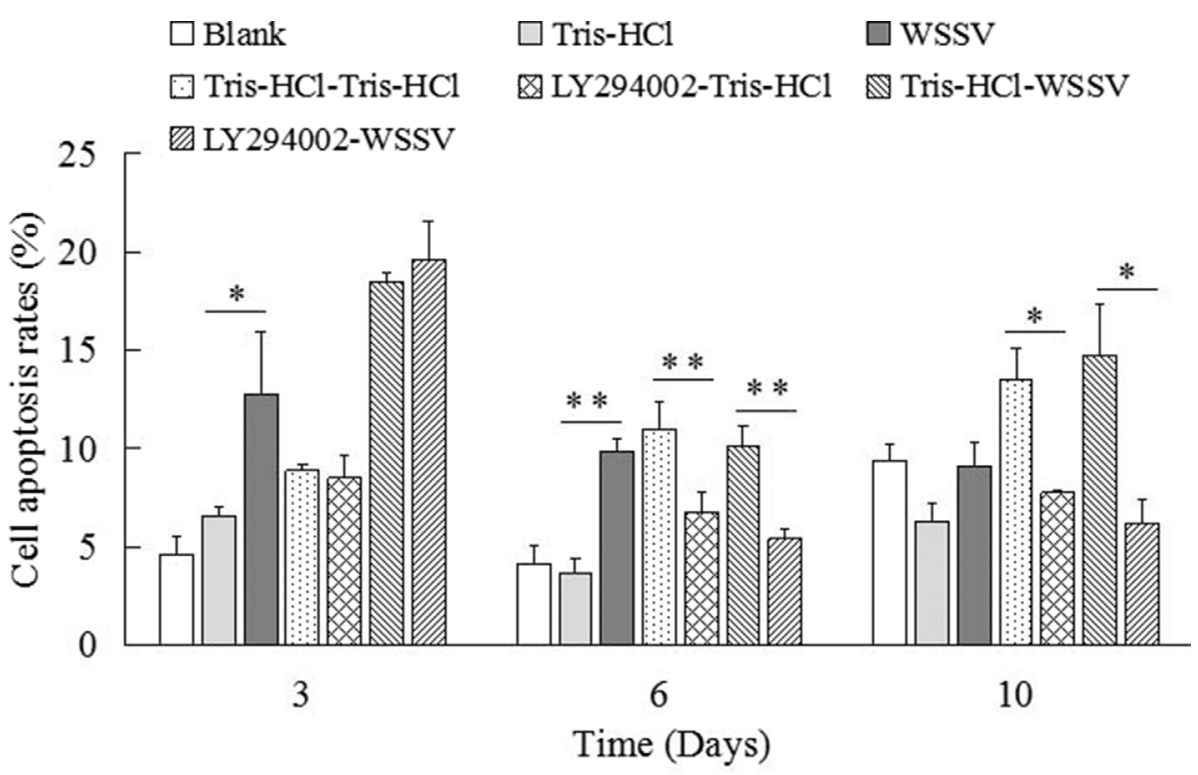

Fig. 3. Flow cytometry analysis of apoptosis in semigranular cells (SGC) of seven groups (Blank, Tris-HCl, WSSV, Tris-HCl-Tris-HCl, LY294002-Tris-HCl, Tris-HClWSSV and LY294002-WSSV). The rates of apoptotic cells are shown. Vertical bars represent the mean $\pm \operatorname{SD}(N=5)$. Significant differences between Tris- $\mathrm{HCl}$ and WSSV, Tris$\mathrm{HCl}-\mathrm{Tris}-\mathrm{HCl}$ and LY294002-Tris-HCl, Tris-HCl-WSSV and LY294002-WSSV are indicated with one asterisk at $P<0.05$ and two asterisks at $P<0.01$.

\subsection{The Bax inhibitor-1 mRNA expression in haemocytes of crayfish}

The Bax inhibitor-1 mRNA expression was significantly increased in haemocytes of crayfish after WSSV infection on the third and sixth day (Fig. 6). The relative expression levels of Bax inhibitor-1 in WSSV group on the third and sixth day were up-regulated, and 3.73-fold and 3.95fold higher $(P<0.05)$ than that of Tris-HCl group, respectively.

The Bax inhibitor-1 mRNA expression levels in LY294002 pretreatment group were significantly lower than those in Tris-HCl pretreatment crayfish on the third day regardless whether the crayfish were infected with WSSV (Fig. 6). The Bax inhibitor-1 level in LY294002-Tris-HCl group was 0.42 -fold lower $(P<0.01)$ than that in Tris-HCl-Tris-HCl group, and Bax inhibitor-1 level in LY294002-WSSV group was 0.33 -fold lower $(P<0.05)$ than that of Tris-HCl-WSSV group on the third day.

\subsection{The Toll mRNA expression in haemocytes of crayfish}

The Toll mRNA expression was also decreased in LY294002-Tris$\mathrm{HCl}$ group compared to that in Tris- $\mathrm{HCl}-\mathrm{Tris}-\mathrm{HCl}$ group on the third, sixth and tenth day (Fig. 7). The Toll mRNA expression in LY294002-

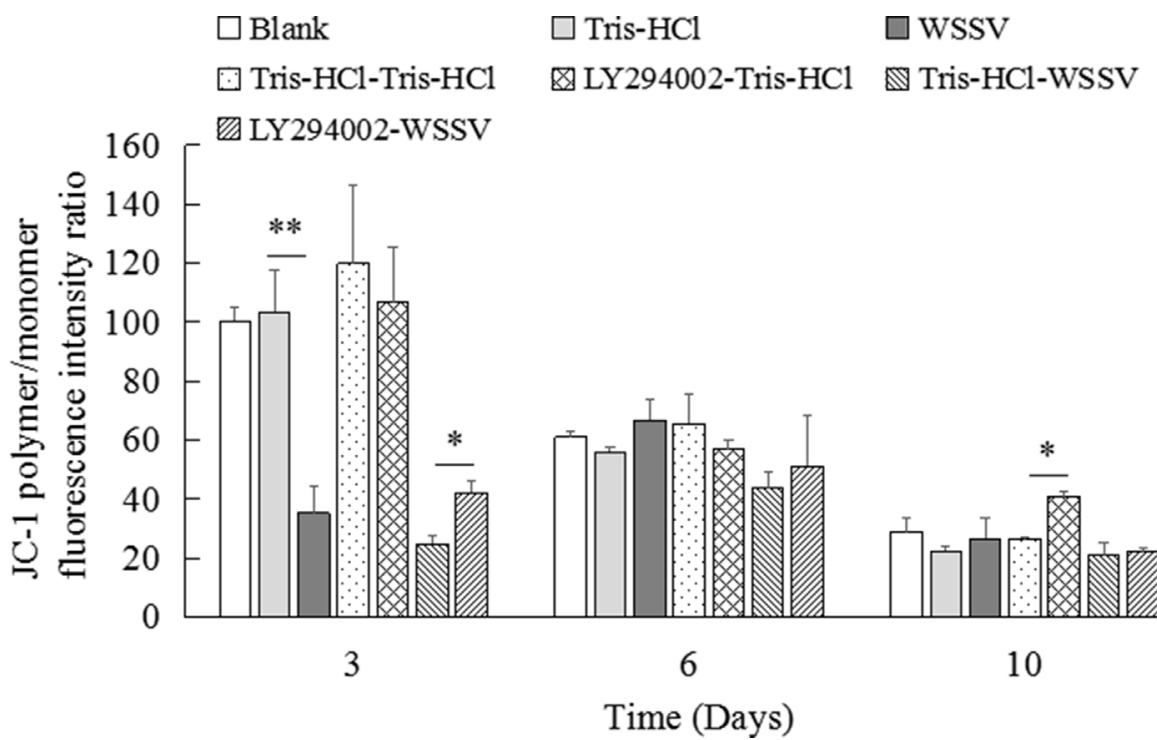

Fig. 4. The $\Delta \psi$ were evaluated by the JC-1 polymer/ monomer fluorescence intensity ratio in the seven groups (Blank, Tris-HCl, WSSV, Tris-HCl-Tris-HCl, LY294002-Tris$\mathrm{HCl}$, Tris-HCl-WSSV and LY294002-WSSV) on the third, sixth and tenth day. Each symbol and vertical bars represented the mean \pm SD $(N=5)$. Significant differences between Tris- $\mathrm{HCl}$ and WSSV, Tris-HCl-Tris- $\mathrm{HCl}$ and LY294002-Tris-HCl, Tris-HCl-WSSV and LY294002-WSSV were indicated with one asterisk at $P<0.05$ and two asterisks at $P<0.01$. 


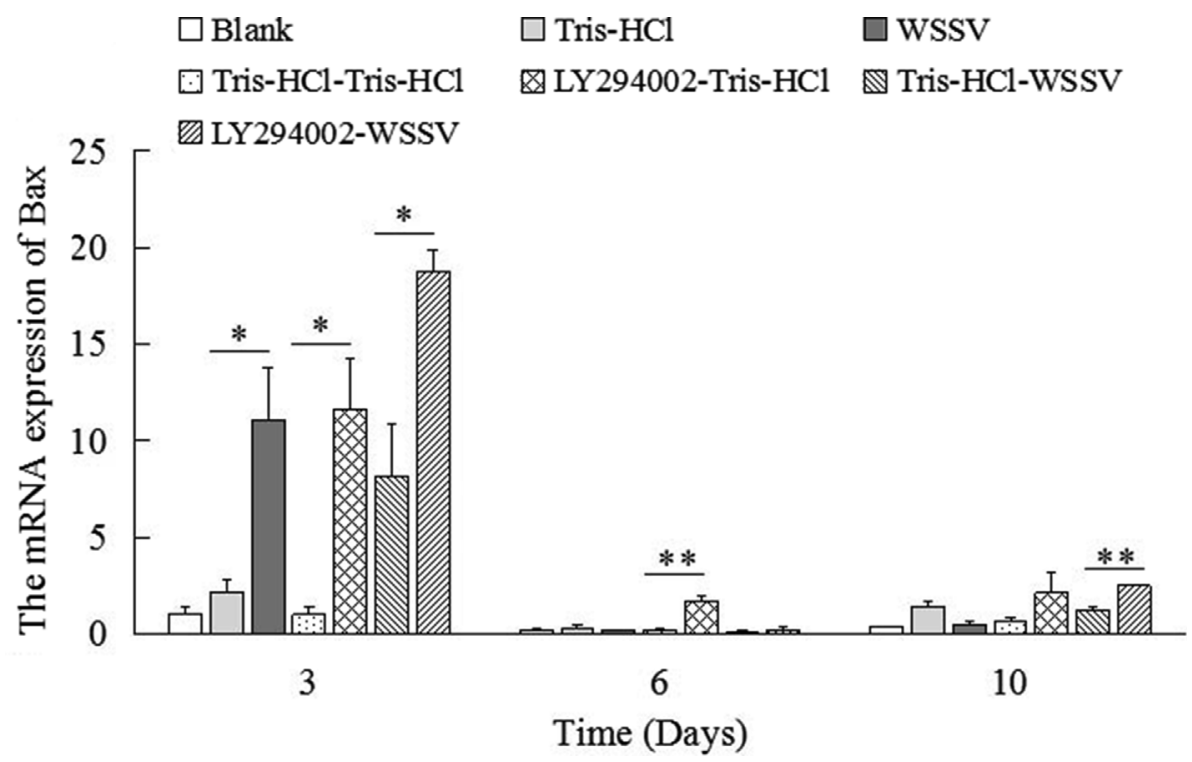

Fig. 5. The quantification of Bax transcript relative to $18 \mathrm{~s}$ RNA transcript in the seven groups (Blank, Tris-HCl, WSSV, Tris-HCl-Tris-HCl, LY294002-Tris-HCl, Tris-HCl-WSSV and LY294002-WSSV) on the third, sixth and tenth day. Each symbol and vertical bars represented the mean \pm SD $(N=5)$. Significant differences between Tris- $\mathrm{HCl}$ and WSSV, Tris-HCl-Tris-HCl and LY294002-Tris-HCl, Tris-HClWSSV and LY294002-WSSV were indicated with one asterisk at $P<0.05$ and two asterisks at $P<0.01$.

\section{Discussion}

Activation of the PI3K/AKT signaling pathway is common to viral infection, and many virus manipulated this signaling pathway to ensure successful virus replication. The blockage of PI3K/AKT signaling pathway would inhibit viral replication, such as CMV and PRRSV $[7,9]$. Liu and Cohen demonstrated that inhibition of PI3K/AKT signaling pathway with LY294002 could delay CMV entry, reduce viral immediate-early and early gene expression, and viral DNA replication [25]. Similarly, a significant reduction in PRRSV virus titers was observed in the culture supernatant, when monocytes derived-dendritic cells were pre-treated with LY294002 followed by PRRSV infection [9]. In the present study, the PI3K inhibitor (LY294002) was able to reduce basal AKT phosphorylation (Fig. 1), which showed that this inhibitor was affecting the activity of PI3K in the haemocytes of crayfish. To further test whether PI3K/AKT signaling pathway was involved in WSSV replication, we treated crayfish with LY294002 and detected the WSSV copy numbers after WSSV infection. The results indicated that the WSSV copy numbers in crayfish pretreated with LY294002 were significantly lower than those in Tris- $\mathrm{HCl}$ pretreatment crayfish on the sixth and tenth day post WSSV infection (Fig. 2), which indicated that blockage of the PI3K/AKT signaling pathway could inhibit WSSV replication in crayfish. Viruses are to exploit and manipulate the host pathways to establish an environment favorable for their replication and persistence infection [14]. We demonstrated that the host PI3K/ AKT signaling pathway play positive roles in WSSV replication.

During the co-evolution of virus and host, apoptosis was employed by the host as one of the protective measures to prevent and promote virus clearance, which is also utilized for viruses to evade the host immune system to ensure viral replication [26]. In the present study, there were apoptosis rates of $12.80 \pm 3.10 \%$ and $9.87 \pm 0.68 \%$ on the third and sixth day post WSSV infection, which were 1.94-fold $(P<0.05)$ and 2.69 -fold $(P<0.01)$ higher than the Tris-HCl group in SGC of crayfish, respectively (Fig. 3). In mitochondrial permeability transition (MPT), the inner and outer mitochondrial membranes communicate via high-conductance channels that dissipate $\Delta \psi$ and release cytochrome c. Induction of MPT can be caused by diverse stimuli and is believed to be involved in cell death [27]. In SGC stained with JC-1,

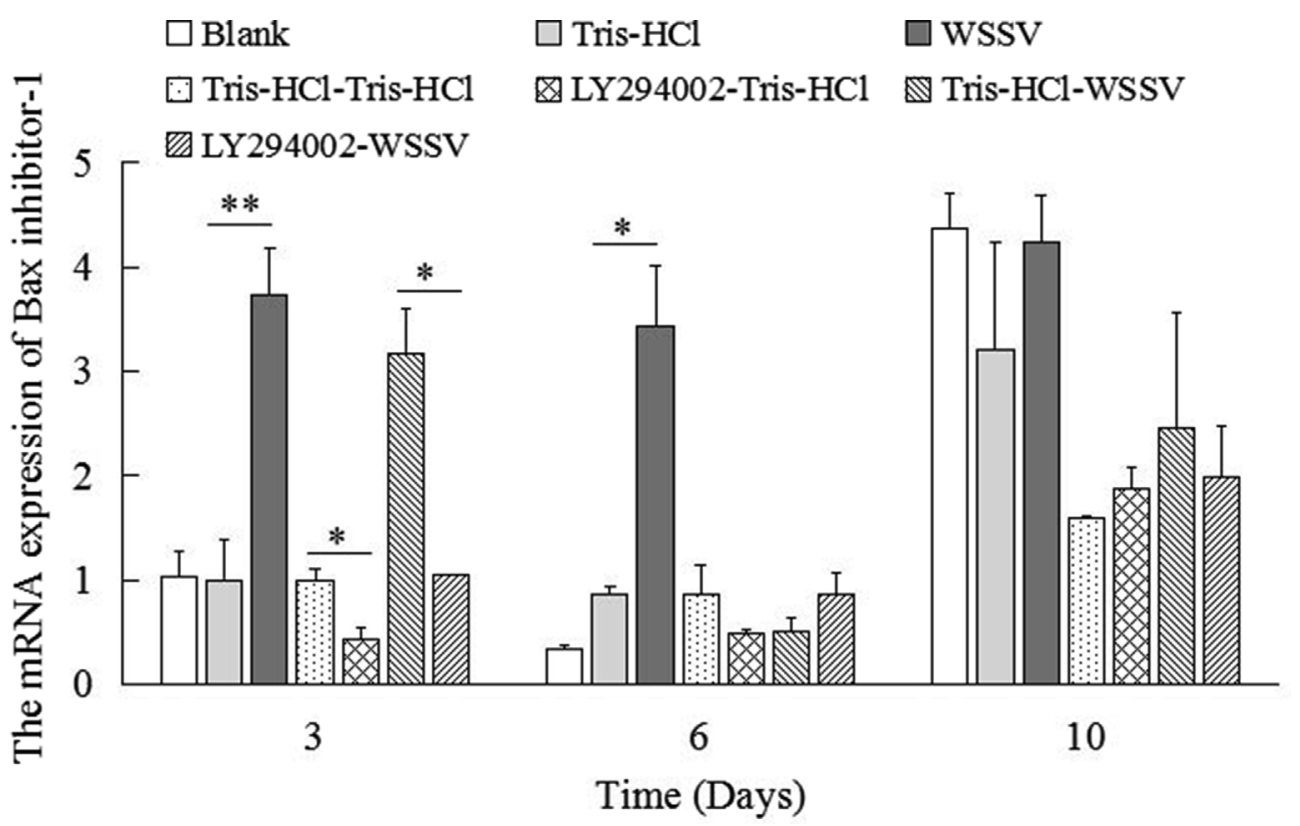

Fig. 6. The quantification of Bax inhibitor-1 transcript relative to 18s RNA transcript in the seven groups (Blank, Tris-HCl, WSSV, Tris-HClTris-HCl, LY294002-Tris-HCl, Tris-HCl-WSSV and LY294002-WSSV) on the third, sixth and tenth day. Each symbol and vertical bars represented the mean $\pm \mathrm{SD}(N=5)$. Significant differences between Tris- $\mathrm{HCl}$ and WSSV, Tris-HCl-Tris- $\mathrm{HCl}$ and LY294002-Tris-HCl, Tris-HCl-WSSV and LY294002-WSSV were indicated with one asterisk at $P<0.05$ and two asterisks at $P<0.01$. 


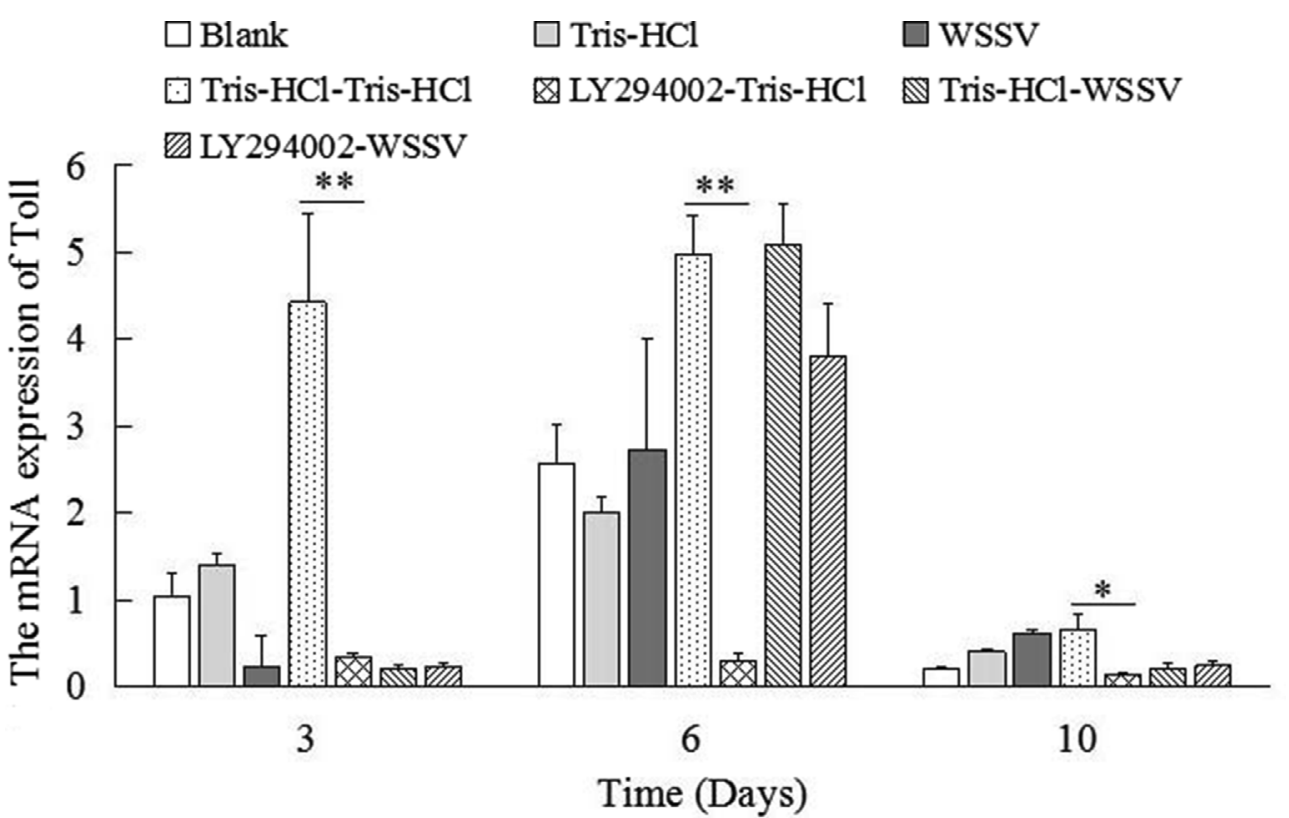

Fig. 7. The quantification of Toll transcript relative to 18s RNA transcript in the seven groups (Blank, Tris- $\mathrm{HCl}$, WSSV, Tris-HCl-Tris-HCl, LY294002-Tris-HCl, Tris-HCl-WSSV and LY294002-WSSV) on the third, sixth and tenth day. on the third, sixth and tenth day. Each symbol and vertical bars represented the mean $\pm \mathrm{SD}(N=5)$. Significant differences between Tris-HCl-Tris- $\mathrm{HCl}$ and LY294002-Tris-HCl were indicated with one asterisk at $P<0.05$ and two asterisks at $P<0.01$. there was lower $\Delta \psi(P<0.01)$ in crayfish post WSSV infection compared to that in crayfish after Tris- $\mathrm{HCl}$ injection on the third day (Fig. 4). The result demonstrated that WSSV induced a stress response (SGC apoptosis), which might participate in the maintenance of the long-term WSSV infection and decline of the virus clearance. Targeting the PI3K/AKT pathway is a notable strategy taken by various viruses to inhibit apoptosis. For example, inhibition of PI3K activation with LY294002 could increase the cleavage and release of caspase-3, and induce apoptosis during largemouth Bass virus (LMBV), dengue virus (DEN) or Japanese encephalitis virus (JEV) infection [11-13]. However, our findings are in conflict with these results, which have suggested that PI3K inhibition is required to block apoptosis in WSSV-infected crayfish (Figs. 3 and 4). Similarly, in herpes simplex virus-1 (HSV-1)-infected OC3 cells, LY294002 treatment did not increase apoptosis and affect cell viability [28]. Therefore, the relationship between PI3K activity and apoptosis may vary in different host or virus strains. WSSV infection leads to the outbreak death of cultured Prawn in a short time, but crayfish is resistant and tolerance to WSSV infection. Therefore, the balance between the pro- and anti-apoptosis activation processes regulated by PI3K/AKT signaling pathway would determine the fate of the WSSV-infected host.

Bax, a pro-apoptotic member of the Bcl-2 family, localizes in the cytoplasm but redistributes to mitochondria in response to apoptotic stimuli, where it induces cytochrome release [29]. The PI3K/AKT signaling pathway plays a pivotal role in the regulation of apoptosis, and AKT activates translocation of Bax from the cytoplasm to the mitochondria [30]. In order to investigate the mechanism of apoptosis in WSSV infected crayfish, Bax and Bax inhibitor-1 were examined. The expression level of Bax mRNA was increased on the third day after WSSV infection, which indicated that WSSV induced Bax production and Bax redistributed in the mitochondria, resulting in cytochrome release and apoptosis. A significant increase of Bax expression level in LY294002 pretreatment crayfish and a significantly lower proportion of apoptosis cells were observed (Figs. 3 and 4). LY294002 treatment increased Bax expression level, but it might block the effect of WSSV to activate Bax translocation to the mitochondria and be anti-apoptosis

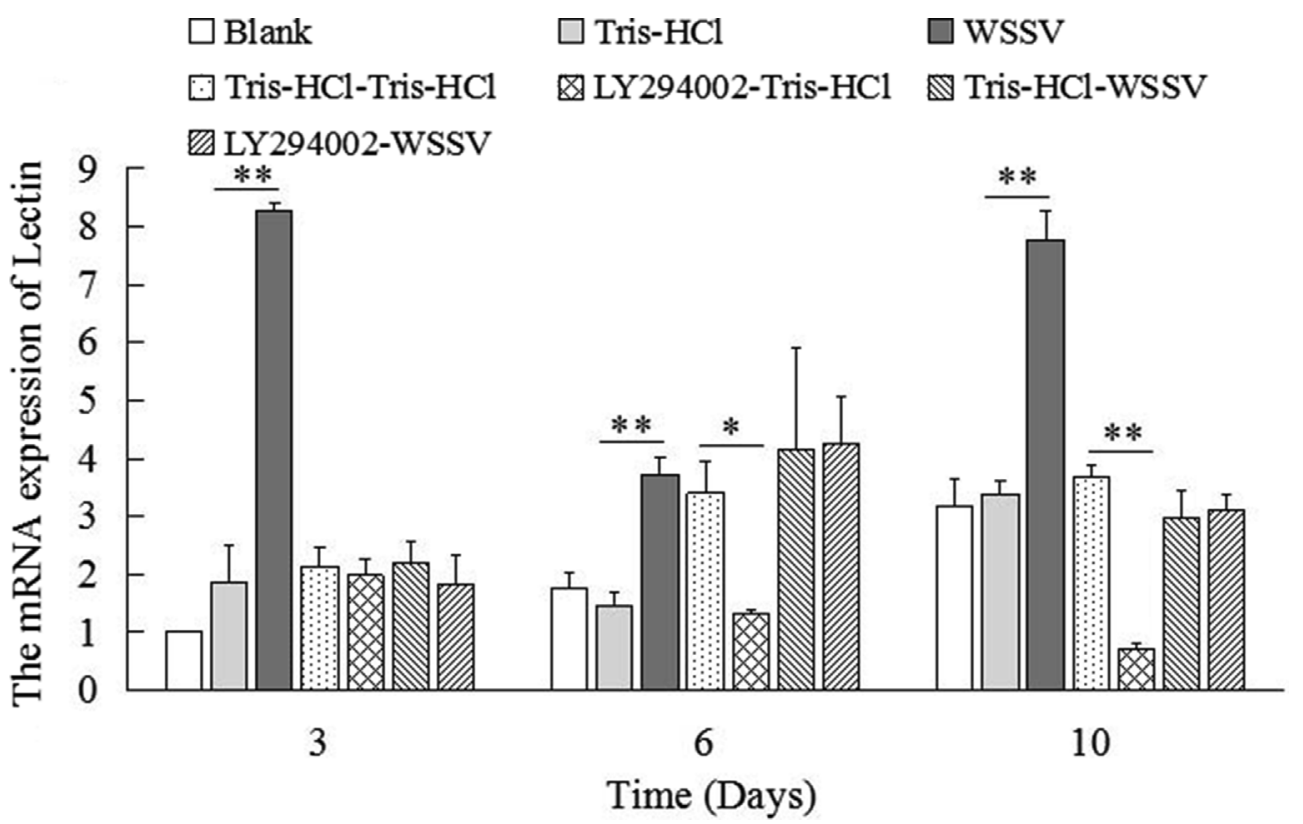

Fig. 8. The quantification of lectin transcript relative to 18s RNA transcript in the seven groups (Blank, Tris- $\mathrm{HCl}$, WSSV, Tris-HCl-Tris-HCl, LY294002-Tris-HCl, Tris-HCl-WSSV and LY294002-WSSV) on the third, sixth and tenth day. Each symbol and vertical bars represented the mean $\pm \mathrm{SD}(N=5)$. Significant differences between Tris- $\mathrm{HCl}$ and WSSV, Tris-HCl-Tris-HCl and LY294002-Tris- $\mathrm{HCl}$ were indicated with one asterisk at $P<0.05$ and two asterisks at $P<0.01$. 
activation processes. Crayfish Bax inhibitor-1 protein has been proven as an anti-apoptotic factor, which suppresses programmed cell death and is beneficial for WSSV replication [31]. The Bax inhibitor-1 levels in LY294002 pretreatment group were significantly lower than those in Tris- $\mathrm{HCl}$ pretreatment crayfish on the third day regardless whether the crayfish were infected with WSSV (Fig. 6). Bax inhibitor-1 protein neutralizes, balances Bax activity and plays a protective function as the main suppressor of Bax-induced cell death in environmental responses [32]. The results together demonstrated the PI3K/AKT signaling pathway played an important role in the regulation of host cell survival through regulation of Bax expression levels.

Toll-like receptors (TLRs), a major family of PRRs, play an important role in innate host defense as well as in initiation of adaptive immune responses. PI3K inhibitors (i.e., wortmannin and LY294.002) could modulate TLR-induced cytokine production and enhance antitumor immunity in humans. In addition, previous studies implicated PI3K and its substrate AKT in the regulation of TLR signaling. In the present study, the Toll mRNA expression was significantly down-regulated in LY294002 pretreatment crayfish on the third, sixth and tenth day (Fig. 7), which indicated that PI3K signaling played a major role in inducing the up-regulation of Toll expression and activating TLR signaling. Lectin proteins family is one of the important components in the immune defense system of invertebrate. The lectin mRNA expression was significantly increased in haemocytes of crayfish after WSSV infection on the third, sixth and tenth day, indicating lectin involved in the immune defense responses against WSSV in crayfish. There was a significant decrease lectin expression level in LY294002-Tris-HCl group compared to that in Tris-HCl-Tris-HCl group on the sixth and tenth day (Fig. 8). The lectin expression in LY294002 pretreatment crayfish was totally consistent with the previous Toll expression. The PI3K/AKT signaling is involved in the regulation of Toll and lectin signaling outcomes, which induced and regulated innate immune response in crayfish.

In conclusion, the PI3K/AKT signaling pathway play positive roles in WSSV replication. Blockage of the PI3K/AKT signaling pathway could decrease the WSSV copy numbers and host cellular apoptosis in WSSV-infected crayfish. Furthermore, the PI3K/AKT signaling pathway could regulate the apoptosis-related factors of Bax and Bax-inhibitor-1, as well as the level of innate immune defense protein (Toll and lectin).

\section{Acknowledgement}

This research was supported by grants from the National Natural Science Foundation of China (No. 31402330) and (No. 41576106), the Youth Prominent Teacher Training Plan of Binzhou Medical University and the Youth Innovation Promotion Association of CAS (2014190).

\section{References}

[1] T.D. Troutman, W. Hu, S. Fulenchek, T. Yamazaki, T. Kurosaki, J.F. Bazan, C. Pasare, Role for B-cell adapter for PI3K (BCAP) as a signaling adapter linking Toll-like receptors (TLRs) to serine/threonine kinases PI3K/AKT, Proc. Natl. Acad. Sci. U. S. A. 109 (2012) 273-278.

[2] L. Reshi, J.L. Wu, H.V. Wang, J.R. Hong, Aquatic viruses induce host cell death pathways and its application, Virus Res. 211 (2016) 133-144.

[3] M.F. Saeed, A.A. Kolokoltsov, A.N. Freiberg, M.R. Holbrook, R.A. Davey, Phosphoinositide-3 kinase-AKT pathway controls cellular entry of Ebola virus, PLoS Pathog. 4 (2008) e1000141.

[4] F. François, M.E. Klotman, Phosphatidylinositol 3-kinase regulates human immunodeficiency virus type 1 replication following viral entry in primary CD $4+T$ lymphocytes and macrophages, J. Virol. 77 (2003) 2539-2549.

[5] S.Z. Feng, W.S. Cao, M. Liao, The PI3K/AKT pathway is involved in early infection of some exogenous avian leukosis viruses, J. Gen. Virol. 92 (2011) 1688-1697.

[6] M.J. Hsu, C.Y. Wu, H.H. Chiang, Y.L. Lai, S.L. Hung, PI3K/AKT signaling mediated apoptosis blockage and viral gene expression in oral epithelial cells during herpes simplex virus infection, Virus Res. 153 (2010) 36-43.

[7] R.A. Johnson, X. Wang, X.L. Ma, S.M. Huong, E.S. Huang, Human cytomegalovirus up-regulates the phosphatidylinositol3-kinase (PI3-K) pathway: inhibition of PI3-K activity inhibits viral replication and virus-induced signaling, J. Virol. 75 (2001)
6022-6032.

[8] O. Du, Y. Huang, T. Wang, X. Zhang, Y. Chen, B, Cui, D, Li, X. Zhao, W. Zhang, L. Chang, D. Tong, Porcine circovirus type 2 activates PI3K/AKT and p38 MAPK pathways to promote interleukin-10 production in macrophages via Cap interaction of gC1qR, Oncotarget 7 (2016) 17492-17507.

[9] S. Pujhari, M. Kryworuchko, Zakhartchouk AN Role of phosphatidylinositol-3-kinase (PI3K) and the mammaliantarget of rapamycin (mTOR) signalling pathways in porcine reproductive and respiratory syndrome virus (PRRSV) replication, Virus Res. 194 (2014) 138-144.

[10] D. Jackson, M.J. Killip, C.S. Galloway, R.J. Russell, R.E. Randall, Loss of function of the influenza A virus NS1 protein promotes apoptosis but this is not due to a failure to activate phosphatidylinositol 3-kinase (PI3K), Virology 396 (2010) 94-105.

[11] X. Huang, W. Wang, Y. Huang, L. Xu, Q. Qin, Involvement of the PI3K and ERK signaling pathways in largemouth bass virus-induced apoptosis and viral replication, Fish Shellfish Immunol. 41 (2014) 371-379.

[12] Y. Liu, H. Liu, J. Zou, B. Zhang, Z. Yuan, Dengue virus subgenomic RNA induces apoptosis through the Bcl-2-mediated PI3k/AKT signaling pathway, Virology 448 (2014) 15-25.

[13] T.H. Chang, C.L. Liao, Y.L. Lin, Flavivirus induces interferon-beta gene expression through a pathway involving RIG-I-dependent IRF-3 and PI3K-dependent NFkappaB activation, Microb. Infect. 8 (2006) 157-171.

[14] B. Verbruggen, L.K. Bickley, R. van Aerle, K.S. Bateman, G.D. Stentiford, E.M. Santos, C.R. Tyler, Molecular mechanisms of white spot syndrome virus infection and perspectives on treatments, Viruses 8 (2016) 23.

[15] F.H. Yuan, Y.G. Chen, Z.Z. Zhang, H.T. Yue, H.T. Bi, K. Yuan, S.P. Weng, J.G. He, Y.H. Chen, Down-regulation apoptosis signal-regulating kinase 1 gene reduced the Litopenaeus vannamei hemocyte apoptosis in WSSV infection, Fish Shellfish Immunol. 50 (2016) 109-116.

[16] K. Wongprasert, K. Khanobdee, S.S. Glunukarn, P. Meeratana, B. Withyachumnarnkul, Time-course and levels of apoptosis in various tissues of black tiger shrimp Penaeus monodon infected with white-spot syndrome virus, Dis. Aquat. Org. 55 (2003) 3-10.

[17] Y. Zhang, L. Song, J. Zhao, L. Wang, P. Kong, L. Liu, M. Wang, L. Qiu, Protective immunity induced by CpG ODNs against white spot syndrome virus (WSSV) via intermediation of virus replication indirectly in Litopenaeus vannamei, Dev. Comp. Immunol. 34 (2010) 418-424.

[18] J.F. Lan, S. Wei, Y.Q. Wang, Y.J. Dai, J.G. Tu, L.J. Zhao, X.C. Li, Q.W. Qin, N. Chen, L. Lin, PcToll3 was involved in anti-Vibrio response by regulating the expression of antimicrobial peptides in red swamp crayfish, Procambarus clarkii, Fish Shellfish Immunol. 57 (2016) 17-24.

[19] Y. Dai, Y. Wang, L. Zhao, Z. Qin, J. Yuan, Q. Qin, L. Lin, J. Lan, A novel L-type lectin was required for the multiplication of WSSV in red swamp crayfish (Procambarus clakii), Fish Shellfish Immunol. 55 (2016) 48-55.

[20] J. Feng, L. Zhao, M. Jin, T. Li, L. Wu, Y. Chen, Q. Ren, Toll receptor response to white spot syndrome virus challenge in giant freshwater prawns (Macrobrachium rosenbergii), Fish Shellfish Immunol. 57 (2016) 148-159.

[21] Y. Tian, T. Chen, W. Huang, P. Luo, D. Huo, L. Yun, C. Hu, Y. Cai, A new L-type lectin (LvLTLC1) from the shrimp Litopenaeus vannamei facilitates the clearance of Vibrio harveyi, Fish Shellfish Immunol. 73 (2018) 185-191.

[22] Y. Zhang, H. Sun, H. Zhao, X. Chen, J. Li, B. Li, Early apoptosis of monocytes induced by Helicobacter pylori infection through multiple pathways, Dev. Comp. Immunol. 73 (2017) 46-51.

[23] J. Xian, A. Wang, Y. Miao, Application of flow cytometry in studies of classification, viability and immune parameters of Procambarus clarkii haemocytes, Freshw. Fish. 42 (2012) 9-14.

[24] Y. Zhang, L. Qiu, L. Song, H. Zhang, J. Zhao, L. Wang, Y. Yu, C. Li, F. Li, K. Xing, B. Huang, Cloning and characterization of a novel C-type lectin gene from shrimp Litopenaeus vannamei, Fish Shellfish Immunol. 26 (2009) 183-192.

[25] X. Liu, J.I. Cohen, The role of PI3K/AKT in human herpesvirus infection: from the bench to the bedside, Virology 479-480 (2015) 568-577.

[26] E.W. Sun, Y.F. Shi, Apoptosis: the quiet death silences the immune system, Pharmacol. Ther. 92 (2001) 135-145.

[27] G.A. dos Santos, R.S. Abreu e Lima, C.R. Pestana, A.S. Lima, P.S. Scheucher, C.H. Thomé, H.L. Gimenes-Teixeira, B.A. Santana-Lemos, A.R. Lucena-Araujo, F.P. Rodrigues, R. Nasr, S.A. Uyemura, R.P. Falcão, H. de Thé, P.P. Pandolfi, C. Curti, E.M. Rego, $(+) \alpha$-Tocopheryl succinate inhibits the mitochondrial respiratory chain complex I and is as effective as arsenic trioxide or ATRA against acute promyelocytic leukemia in vivo, Leukemia 26 (2012) 451-460.

[28] M.J. Hsu, C.Y. Wu, H.H. Chiang, Y.L. Lai, S.L. Hung, PI3K/AKT signaling mediated apoptosis blockage and viral gene expression in oral epithelial cells during herpes simplex virus infection, Virus Res. 153 (2010) 36-43.

[29] Y. Cui, P. Lu, G. Song, Q. Liu, D. Zhu, X. Liu, Involvement of PI3K/AKT, ERK and p38 signaling pathways in emodin-mediated extrinsic and intrinsic human hepatoblastoma cell apoptosis, Food Chem. Toxicol. 92 (2016) 26-37.

[30] F. Tsuruta, N. Masuyama, Y. Gotoh, The phosphatidylinositol 3-kinase (PI3K)-AKT pathway suppresses Bax translocation to mitochondria, J. Biol. Chem. 277 (2002) 14040-14047.

[31] Z.Q. Du, J.F. Lan, Y.D. Weng, X.F. Zhao, J.X. Wang, BAX inhibitor-1 silencing suppresses white spot syndrome virus replication in red swamp crayfish, Procambarus clarkii, Fish Shellfish Immunol. 35 (2013) 46-53.

[32] M. Kawai, L. Pan, J.C. Reed, H. Uchimiya, Evolutionally conserved plant homologue of the Bax inhibitor-1 (BI-1) gene capable of suppressing Bax-induced cell death in yeast(1), FEBS Lett. 464 (1999) 143-147. 